\title{
REGULARITY IN KINETIC FORMULATIONS VIA AVERAGING LEMMAS
}

\author{
Pierre-Emmanuel Jabin ${ }^{1}$ and Benoît Perthame ${ }^{1}$
}

\begin{abstract}
We present a new class of averaging lemmas directly motivated by the question of regularity for different nonlinear equations or variational problems which admit a kinetic formulation. In particular they improve the known regularity for systems like $\gamma=3$ in isentropic gas dynamics or in some variational problems arising in thin micromagnetic films. They also allow to obtain directly the best known regularizing effect in multidimensional scalar conservation laws. The new ingredient here is to use velocity regularity for the solution to the transport equation under consideration. The method of proof is based on a decomposition of the density in Fourier space, combined with the $K$-method of real interpolation.
\end{abstract}

Mathematics Subject Classification. 35L65, 35B30, 35B65, 74G65, 83D30.

Received December 3, 2001. Revised February 2, 2002.

\section{INTRODUCTION}

Kinetic formulations allow to consider nonlinear problems (balance laws or variational problems) and, using a nonlinear function $f$ of the unknown, to transform these problems in a singular linear transport equation on $f$. The simplest example is that of the entropy solution $u \in C\left(\mathbb{R}^{+} ; L^{1}\left(\mathbb{R}^{d}\right)\right)$ to a multidimensional scalar conservation law

$$
\begin{aligned}
& \partial_{t} u(t, x)+\operatorname{div} A(u)=0, \quad t>0, x \in \mathbb{R}^{d}, \\
& \partial_{t} S(u(t, x))+\operatorname{div} \eta^{S}(u) \leq 0,
\end{aligned}
$$

for all convex function $S(\cdot)$ with $S(0)=0$ and using the notations $\eta^{S}(u)=\int_{0}^{u} S^{\prime}(\cdot) a(\cdot), a=A^{\prime}: \mathbb{R} \rightarrow \mathbb{R}^{d}$. Then, we define, for $v \in \mathbb{R}$, the "equilibrium" function $f(t, x, v)$ thanks to

$$
f(t, x, v)=\left\{\begin{array}{cl}
+1, & \text { for } 0<v<u(t, x) \\
-1, & \text { for } u(t, x)<v<0 \\
0, & \text { otherwise. }
\end{array}\right.
$$

The theory of kinetic formulations states that (1.1) is equivalent to write the kinetic equation on $f$

$$
\partial_{t} f+a(v) \cdot \nabla_{x} f=\partial_{v} m(t, x, v)
$$

Keywords and phrases: Regularizing effects, kinetic formulation, averaging lemmas, hyperbolic equations, line-energy GinzburgLandau.

1 École Normale Supérieure, Département de Mathématiques et Applications, UMR 8553 du CNRS, 45 rue d'Ulm, 75230 Paris Cedex 05, France; e-mail: jabin@dma.ens.fr, perthame@dma.ens.fr 
for some unknown nonnegative bounded measure $m$. The derivation is obtained by integrating $(1.3)$ against $S^{\prime}(v)$, and since we have

$$
S(u)=\int_{\mathbb{R}} S^{\prime}(v) f(t, x, v) \mathrm{d} v, \quad \eta^{S}(u)=\int_{\mathbb{R}} S^{\prime}(v) a(v) f(t, x, v) \mathrm{d} v,
$$

the kinetic formulation turns out to provide the inequalities

$$
\partial_{t} S(u(t, x))+\operatorname{div} \eta^{S}(u)=-\int_{\mathbb{R}} S^{\prime \prime}(v) m(t, x, v) \mathrm{d} v .
$$

Therefore the inequalities in the second equation of (1.1) are equivalent to the positivity of $m$. Also a control of the total mass of the measure is obtained using $S(v)=v^{2} / 2$ in the above inequality

$$
\int_{0}^{\infty} \int_{\mathbb{R} \times \mathbb{R}^{d}} m(t, x, v) \mathrm{d} t \mathrm{~d} v \mathrm{~d} x \leq \frac{1}{2}\left\|u^{0}\right\|_{L^{2}\left(\mathbb{R}^{d}\right)}^{2} .
$$

This method turns out to provide a tool for studying regularizing effects for the hyperbolic equation (1.1), when a non-degeneracy condition on the fluxes $A$ is satisfied. Indeed, averaging lemmas may be applied to the linear transport equation (1.3) and provide regularity in low order Sobolev spaces (it is known that BV regularity is the best that can be expected) for averages like (1.5) below.

Several other examples of such a kinetic formulation, and of related regularizing effects, have been derived and are recalled below.

The purpose of this paper is to show that the known regularizing effects for these examples can be improved using an additional information on the function $f$, namely its $v$ regularity. This motivates to first study a new class of averaging lemmas.

In the end of this introduction, we first state our averaging results and then we give three examples of applications to regularizing effects.

In the second and third sections, we prove the results and in a last section we treat cases (higher $v$ derivability on $f$ ) which require another method of proof.

\subsection{The averaging results}

We consider the following equation

$$
v \cdot \nabla_{x} f=\Delta_{x}^{\alpha / 2} g, \quad x \in \mathbb{R}^{d}, v \in \mathbb{R}^{d}
$$

Now we choose any $\phi \in C_{c}^{\infty}\left(\mathbb{R}^{d}\right)$ and define

$$
\rho(x)=\int_{\mathbb{R}^{d}} f(x, v) \phi(v) \mathrm{d} v .
$$

Assume that

$$
\begin{aligned}
& g \in L^{p}\left(\mathbb{R}^{d}, W_{v}^{\beta, p}\left(\mathbb{R}^{d}\right)\right), \quad 1<p \leq 2, \beta \leq \frac{1}{2} \\
& f \in L^{q}\left(\mathbb{R}^{d}, W_{v}^{\gamma, q}\left(\mathbb{R}^{d}\right)\right), \quad 1<q \leq 2,1-\frac{1}{q}<\gamma \leq \frac{1}{2} .
\end{aligned}
$$

We also point out that the results and proofs extend in the same way for exponents $p$ or $q$ larger than 2 . Then, we have to replace $p$ and $q$ by $\min (p, \bar{p})$ and $\min (q, \bar{q})$ in formula $(1.7)$ below $(\bar{p}$ denotes here the conjugate exponent to $p$ ). It is also possible to deal with exponents $\beta$ or $\gamma$ larger than $1 / 2$ but the question is more delicate. We restrict ourselves to $\beta, \gamma<1 / 2$ for the moment and leave the other cases to the last section of the paper. 
As usual for averaging lemmas, we state that the average $\rho$ is in fact more regular than $f$ itself. This can be quantified as follows

Theorem 1.1. (Case $0 \leq \alpha<1$ ) Let $f, g$ satisfy (1.4) and (1.6), then we have for $s^{\prime}<s=\theta(1-\alpha)$ and $r^{\prime}<r$ with $\frac{1}{r}=\frac{\theta}{p}+\frac{1-\theta}{q}$,

$$
\begin{gathered}
\|\rho\|_{W_{l o c}^{s^{\prime}, r^{\prime}}} \leq C\left(\|g\|_{L_{x}^{p} W_{v}^{\beta, p}}+\|f\|_{L_{x}^{q} W_{v}^{\gamma, q}}\right), \\
\text { with } \quad \theta=\frac{1+\gamma-1 / q}{1+\gamma-\beta+1 / p-1 / q} .
\end{gathered}
$$

Remark 1.1. 1. The constant $C$ appearing in Theorem 1.1 depends on the size of the domain on which we estimate $\rho$, on the size of the support of $\phi$ and on its regularity.

2. For $\gamma=0, \beta \leq 0$, we are in a case included in standard averaging lemmas (see in particular [10]). However our result is a bit weaker since it is known in this case that $\rho \in W^{s, r}$ with $s$ and $r$ given by the formulas of Theorem 1.1.

The fact that we do not reach $s^{\prime}=s$ and $r^{\prime}=r$ is due to a choice in our method of proof. We decided, for the sake of simplicity, to reduce it to classical interpolation between $L^{q}$ and $W^{1, p}$ through Lions-Peetre K-method. This looses a little compared to a dyadic decomposition. Nevertheless when $p=q$, the previous theorem may be precised and we obtain $\rho$ in a Besov space but still not the optimal Sobolev space.

Theorem 1.2. (Case $0 \leq \alpha<1, p=q$ ) Let $f, g$ satisfy (1.4) and (1.6) with $p=q$. Assume $\beta, \gamma \leq 0$ or $q=p=2$, then for $s=\theta(1-\alpha)$, $\theta$ given by (1.7), we have

$$
\|\rho\|_{B_{\infty}^{s, p}} \leq C\left(\|g\|_{L_{x}^{p} W_{v}^{\beta, p}}+\|f\|_{L_{x}^{p} W_{v}^{\gamma, p}}\right) .
$$

It is also possible to work with $\alpha=1$ and we obtain:

Theorem 1.3. (Case $\alpha=1$ ) Let $f, g$ satisfy (1.4) and (1.6). Assume $\beta, \gamma \geq 0$, then we have for $\frac{1}{r}=\frac{\theta^{\prime}}{p}+\frac{1-\theta^{\prime}}{q}$ and $\theta^{\prime}<\theta$ given by (1.7) (with equality if $p=q=2$ )

$$
\|\rho\|_{L_{r \infty}} \leq C\|g\|_{L_{x}^{p} W_{v}^{\beta, p}}^{\theta^{\prime}}\|f\|_{L_{x}^{q} W_{v}^{\gamma}, q}^{1-\theta^{\prime}}
$$

Moreover for $p=q$, then for any $\beta, \gamma$ (possibly negative), we have with $\theta^{\prime}<\theta$ given by (1.7) and with equality for $\beta, \gamma \leq 0$ or $p=q=2$

$$
\|\rho\|_{L^{p}} \leq C\|g\|_{L_{x}^{p} W_{v}^{\beta, p}}^{\theta^{\prime}}\|f\|_{L_{x}^{q} W_{v}^{\gamma, q}}^{1-\theta^{\prime}} .
$$

Remark 1.2. 1. Here also the constants $C$ depend on the size of the supports of $f$ and $g$ and on $\phi$.

2. The case $p=q, \beta \leq 0$ and $\gamma=0$ was treated in [24]. We find here almost the same exponent $\theta$ : our exponent is precisely the limit case in [24]. The reason why we obtain it is only because we use product Hardy spaces as in [6] where the limit case was also obtained, our method being exactly the same as in [24] in this case.

3. For $p \neq q$ and $\beta$ or $\gamma$ negative, we are unable to obtain more than the weak inequality

$$
\|\rho\|_{L_{r \infty}} \leq C\left(\|g\|_{L^{p}}+\|f\|_{L^{q}}\right) .
$$

4. The main interest of the theorem is to prove that if a sequence $f_{n}$ satisfies (1.4) with right hand side $g_{n}$ which are compact then $f_{n}$ is also compact. 
To conclude, let us mention that these theorems are also true for the evolution equation

$$
\partial_{t} f+a(v) \cdot \nabla_{x} f=\Delta_{x}^{\alpha / 2} g,
$$

when the field $v \rightarrow a(v)$ satisfies the strongest non degeneracy condition, namely: for all $R>0$, there is a constant $C(R)$ such that for $\xi \in \mathbb{R}^{d}, \tau \in \mathbb{R}$ with $|\xi|+|\tau| \leq 1$, then

$$
\text { meas }\{v \text { s. th. }|v| \leq R, \text { and } \| a(v) . \xi-\tau \mid \leq \varepsilon\} \leq C \varepsilon .
$$

The regularity on the average $\rho$ is then a regularity in time and space but all the formulas given above for the exponents are exactly the same. Weaker non-degeneracy conditions can also be used (see [10,13,24]). Also optimal Sobolev spaces can be reached in some situations (see $[4,8,28]$ ) and better adapted Besov-type spaces can also be used as in [25].

\subsection{Applications to kinetic formulations}

Possible applications include in particular kinetic formulations for scalar conservation laws (see [20] or [15] for the case with dispersion), multibranch systems like isentropic gas dynamics with a pressure law $p(\rho)=\kappa \rho^{3}$ (see $[3,21,27])$ and thin micromagnetic films (see $[1,7,16,17,26]$ ). We refer to the given references and [23] for more details on the kinetic formulations.

Firstly, we wish to give a direct proof of the following:

Theorem 1.4. Let $u(t, x) \in C\left(\mathbb{R}^{+} ; L^{1}\left(\mathbb{R}^{d}\right)\right)$ an entropy solution to a nondegenerate (in the sense of $(1.9)$ ) multidimensional scalar conservation law (1.1), with $u(t=0) \in L^{1} \cap L^{\infty}\left(\mathbb{R}^{d}\right)$, then locally we have

$$
u \in W_{t, x}^{s, r} \quad \text { for all } s<\frac{1}{3}, r<\frac{3}{2} .
$$

This regularity was obtained in [21] with a more complicate argument which involves a bootstrap of averaging lemmas combined with the $L^{1}$ contraction property. Notice that the optimal regularizing effect, from $u^{0} \in L^{\infty}$ to $u(t) \in B V$ is an open question in more than one space dimension. On the other hand two different methods lead to the same exponents $s<\frac{1}{3}$. Also our proof below has he advantage to extend to in homogeneous problems like

$$
\partial_{t} u(t, x)+\operatorname{div} A(u)=f(t, x) \in L^{1}\left(\mathbb{R}^{+} \times \mathbb{R}^{d}\right),
$$

and yields the same regularity. An example in Section 1.3 below shows that the $B V$ regularity fails for this problem.

Proof of Theorem 1.4. We define, for $v \in \mathbb{R}$, the function $f(t, x, v)$ in (1.2) and use its kinetic formulation (1.3). We know that the right hand side is the derivative in $v$ of a bounded Radon measure in $(t, x, v)$. A measure belongs to any Sobolev space $W^{-\alpha,(1-\alpha /(d+2))^{-1}}$ with $\alpha>0$. Therefore, we may also write the equation (1.8), and choose in $(1.4,1.6)$ any $\alpha>0, \beta=-\alpha-1, p=(1-\alpha /(2 d+1))^{-1}$. On the other hand, since the derivative in $v$ of $f$ is a bounded measure and $f$ belongs to $L^{\infty}$, by interpolation we know that $f$ belongs to $L_{t, x}^{q} W_{v}^{\gamma, q}$ for any $q<2$ and $\gamma<\frac{1}{2}$. Applying Theorem 1.1 , with $\gamma \approx \frac{1}{2}, q \approx 2, \alpha \approx 0, p \approx 1, \beta \approx-1$, we immediately deduce the regularity result in Theorem 1.4.

As a second example, we consider a solution $(\rho, \rho u)$ to one dimensional isentropic gas dynamics with a polytropic pressure law $p(\rho)=\kappa \rho^{3}$ (more generally we could consider a multibranch system). This class of systems admits a pure kinetic formulation. As for scalar conservation laws, we may define an equilibrium function $f$ as the indicator function in $v$ of an interval depending on the solution to the system. This function $f$ again satisfies a transport equation (1.8) where the right hand side is now the second derivative in $v$ of a 
bounded measure. By the same method we now apply Theorem 1.1 , with $\gamma \approx \frac{1}{2}, q \approx 2, \alpha \approx 0, p \approx 1, \beta \approx-2$, and obtain that, locally,

$$
u \in W_{t, x}^{s, r} \quad \text { for all } s<\frac{1}{4}, r<\frac{8}{5},
$$

which improves the $W^{1 / 7,7 / 4}$ regularity proved in [20]. Notice that it is an open problem to obtain regularity for other pressure laws.

As a third and final example, we consider a variational problem. In some theories of line energies GinzburgLandau for thin micromagnetic films (see [16] and [26]), it is possible to show that a function $f$ of the magnetization $u(x) \in \mathbb{R}^{2}, x \in \mathbb{R}^{2}$, satisfies the kinetic equation (1.4) in two space dimensions where the right hand side is again the divergence in $v \in \mathbb{R}^{2}$ of a bounded measure. The equilibrium function is defined as $f(x, v)=\mathbb{I}_{u(x) \cdot v>0}$ and thus we are again in the same situation as for scalar conservation laws. We can choose the same parameters in Theorem 1.1 and thus the magnetisation satisfies locally

$$
u \in W_{x}^{s, r} \quad \text { for all } s<\frac{1}{3}, r<\frac{3}{2}
$$

whereas in [16] we had only $W^{1 / 5,5 / 3}$ using the classical averaging lemmas of [10]. Here the $B V$ threshold turns out to be fundamental in the counterexample of [1] and it would be useful to determine the optimal regularity of the solution.

\subsection{Examples and counterexamples of optimality}

We discuss here two examples: for optimality of the averaging result itself and for its application to scalar conservation laws.

We do not know in general if the estimates given in Theorems 1.1, 1.2 and 1.3 are optimal or not, except in the case $\beta=\gamma=1 / 2, p=q=2$. Indeed define $f(x, v)=\mathbb{I}_{u(x) \cdot v>0}$ on $\mathbb{R}^{2} \times \mathbb{R}^{2}$ as in the third example of the previous subsection with $u$ the vortex centered at the origin

$$
u(x)=\frac{x^{\perp}}{|x|}, \quad \forall x \neq 0 .
$$

Then $f$ is solution to the equation (in the theory of line energies Ginzburg-Landau for micromagnetic films, it corresponds to zero energy states, see [17])

$$
v \cdot \nabla_{x} f=0, \quad \forall(x, v) \in \mathbb{R}^{4} .
$$

The function $f$ belongs to $L_{x}^{2} H_{v}^{\beta}$ for any $\beta<1 / 2$ and so does of course the right-hand side, so Theorem 1.1 implies that any moment of $f$ belongs to $H_{\text {loc }}^{s}$ for any $s<1$. We thus obtain that $u$ belongs to $H_{\text {loc }}^{s}$ for any $s<1$, which is the best range of exponent since $u$ does not belong to $H_{\text {loc }}^{1}$.

Next, we discuss optimality for scalar conservation laws. In one dimension, for Burgers-Hopf equation (1.1) with $A(u)=u^{2} / 2$, Oleĭnik's regularizing effect proves that $u$ becomes $B V$ immediately (see [22] and [29] for a multidimension extension). Our result only gives $W_{\mathrm{loc}}^{s, 3 / 2}, s<1 / 3$. Notice however that if we add source terms

$$
\begin{aligned}
& \partial_{t} u+\partial_{x} \frac{u^{2}}{2}=f(t, x), \quad f \in M^{1}\left(\mathbb{R}^{+} \times \mathbb{R}\right), \\
& u(t=0, x)=0,
\end{aligned}
$$

then, Oleĭnik's result and method do not apply. For instance take $f=m \delta(t=0) \delta(x=0)$, the solution $u$ is then

$$
\begin{aligned}
& u(t, x)=\frac{x}{t}, \quad \text { for } 0 \leq x<\sqrt{2 m t}, \\
& u(t, x)=0, \quad \text { for } x<0 \text { or } x \geq \sqrt{2 m t} .
\end{aligned}
$$


Now we superpose such source terms, taking $f=\frac{1}{2 N^{2}} \sum_{i=1}^{N} \sum_{j=1}^{N} \delta(t=i / N) \delta(x=j / N)$. Since the waves interactions are very weak, we infer the following estimate for the Sobolev norm of the solution

$$
\begin{aligned}
\|u\|_{L_{t}^{p} W_{x}^{s, p}} & \geq \int_{0}^{1 / 4} \sum_{j=1}^{N-1} \int_{j / 2 N}^{(j+1) / N} \int_{(j-1) / N}^{j / 2 N} \frac{|u(x)-u(y)|^{p}}{|x-y|^{s p+1}} \mathrm{~d} x \mathrm{~d} y \mathrm{~d} t \\
& \sim \sum_{i=0}^{N / 4} \int_{i / N}^{(i+1) / N} N \times \frac{1}{N^{2}} \times \frac{1}{N^{p}} \times \frac{1}{t^{p / 2}} \times N^{s p+1} \\
& \sim N^{s p-p / 2} .
\end{aligned}
$$

Therefore the norm of $u$ in $L_{t}^{p} W_{x}^{s, p}$ can remain bounded as $N \rightarrow \infty$ only for $s \leq 1 / 2$. Of course $u$ does not belong to $L^{\infty}$ so although the kinetic formulation still applies, $u$ is not an average of $f$ as defined by (1.5) (a cut-off in velocity is needed). However the above estimate remains valid for $u \mathbb{I}_{u<10}$ for instance which is a true average and it is worth noticing that for a function $g \in L_{t, x, v}^{1}$, Theorem 1.1 shows that the average belongs to $W^{s, 1}$ with the same scaling $s<1 / 2.5$

\section{Proof of Theorems $1.1-1.3$}

\subsection{Formulation in Fourier space}

Since we average in velocity against $\phi$, we only have to take into account the velocities in the support of $\phi$ and hence we may assume that $f$ and $g$ have compact support in velocity.

We work in the Fourier space and we denote by $\hat{f}(\xi, v)$ and $\hat{g}(\xi, v)$ the Fourier transforms in the $x$ variable of $f$ and $g$. Equation (1.4) becomes

$$
i \xi \cdot v \hat{f}=|\xi|^{\alpha} \hat{g}
$$

Following the method introduced in [24], we write for any $\lambda>0$ ( $\lambda$ may be very large or very small)

$$
\hat{f}=\frac{\lambda|\xi|}{\lambda|\xi|+i \xi \cdot v} \hat{f}+\frac{|\xi|^{\alpha}}{\lambda|\xi|+i \xi \cdot v} \hat{g}
$$

And thus, we obtain

$$
\rho(x)=T_{1}(f)+T_{2}(g)
$$

with $\left(\mathcal{F}^{-1}\right.$ denoting the inverse Fourier transform)

$$
\begin{aligned}
& T_{1}(f)=\mathcal{F}^{-1}\left(\int_{\mathbb{R}^{d}} \frac{\lambda|\xi|}{\lambda|\xi|+i \xi \cdot v} \hat{f}(\xi, v) \phi(v) \mathrm{d} v\right), \\
& T_{2}(g)=\frac{1}{\lambda} \Delta_{x}^{(\alpha-1) / 2} T_{1}(g) .
\end{aligned}
$$

\subsection{Estimates for the norms of $T_{1}$ in $L^{p}$ spaces}

We now compute the norm of the operators $T_{1}$ (and the norms of $T_{2}$ follows) from $L_{x}^{p}\left(W_{v}^{s, p}\right)$ to $L^{p}$. We begin with the simpler case $p=2$.

Lemma 2.1. for any real number $s, \quad T_{1}: L_{x}^{2}\left(H_{v}^{s}\right) \longrightarrow L^{2}$, with norms

$$
\text { for } \quad s \leq 0, \quad\left\|T_{1}\right\|_{s, 2} \leq C\left(\lambda^{1 / 2+s}+\lambda^{1 / 2}\right),
$$




$$
\begin{array}{cc}
\text { for } 0 \leq s<1 / 2, & \left\|T_{1}\right\|_{s, 2} \leq C(s) \lambda^{1 / 2+s}, \\
\text { for } 1 / 2<s \leq 1, & \left\|T_{1}\right\|_{s, 2} \leq C(s) \lambda .
\end{array}
$$

We give a proof of this lemma below and we first state the general result in more general $L^{p}$ spaces. The proof of the following proposition requires more technical tools and it is given in next section.

Proposition 2.1. $\forall 1<p \leq 2, \quad T_{1}: L_{x}^{p}\left(W_{v}^{s, p}\right) \rightarrow L_{x}^{p}, \quad$ with norm

$$
\begin{aligned}
& \left\|T_{1}\right\|_{s, p} \leq C\left(\lambda^{s+1-1 / p}+\lambda^{1-1 / p}\right), \quad \text { for } s \leq 0, \\
& \left\|T_{1}\right\|_{s, p} \leq C \lambda^{r}, \quad r<r^{*}=s+1-\frac{1}{p}\left(r=r^{*} \text { for } p=2\right), \quad \text { for } 0<s<1 / 2, \\
& \left\|T_{1}\right\|_{s, p} \leq C \lambda^{r}, \quad r<r^{*}=2-s+2 \frac{s-1}{p}\left(r=r^{*} \text { for } p=2\right), \quad \text { for } s>1 / 2 .
\end{aligned}
$$

Proof of Lemma 2.1. The Fourier transform in $v$, for a fixed $\xi \neq 0$, of $\lambda|\xi| /(\lambda|\xi|+i \xi \cdot v)$ is exactly

$$
C \lambda \mathrm{e}^{-\lambda \zeta \cdot \frac{\xi}{|\xi|}} H\left(\zeta \cdot \frac{\xi}{|\xi|}\right) \delta_{\xi}(\zeta)
$$

where $C$ is a given constant independent of $\lambda, H$ is the heavy side step function and $\delta_{\xi}$ is the Dirac mass on the line parallel to $\xi$. We choose a function $\psi \in C_{c}^{\infty}\left(\mathbb{R}^{d-1}\right)$ such that $\psi\left(P R_{\xi} v\right) \phi(v)=\phi(v)$ with $P$ the projection on the hyperplane $v_{1}=0\left(P v=v^{\prime}=\left(v_{2}, \ldots, v_{d}\right)\right)$ and $R_{\xi}$ the rotation such that $R_{\xi} \xi=(|\xi|, 0, \ldots, 0)$. If $\phi$ has compact support in the ball $B(0, R)$ of $\mathbb{R}^{d}$ then for $\psi$ we may take, for instance, any function with constant value 1 in the ball $B(0, R)$ of $\mathbb{R}^{d-1}$.

As a consequence, we claim that for any $0 \leq s<1 / 2$, there is a constant $C$ with

$$
\left\|\frac{\lambda|\xi| \psi\left(P R_{\xi} v\right)}{\lambda|\xi|+i \xi \cdot v}\right\|_{H_{v}^{-s}} \leq C \lambda^{1 / 2+s}
$$

Indeed, since $\xi$ is kept fixed for the moment, we may choose a basis for $v$ where $\vec{v}_{1}$ is parallel to $\xi$. In this case, we have, thanks to the localization in velocity, the inequality

$$
\begin{aligned}
\left\|\frac{\lambda|\xi| \psi\left(P R_{\xi} v\right)}{\lambda|\xi|+i \xi \cdot v}\right\|_{H_{v}^{-s}}^{2} & \leq C \lambda^{2} \int\left(1+|\zeta|^{2}\right)^{-s} \mathrm{e}^{-2 \lambda \zeta_{1}} H\left(\zeta_{1}\right)\left|\hat{\psi}\left(\zeta_{2}, \ldots, \zeta_{d}\right)\right|^{2} \mathrm{~d} \zeta \\
& \leq C \lambda^{2}\left\|\psi\left(v^{\prime}\right)\right\|_{H_{v^{\prime}}^{-s}}^{2} \times \int_{0}^{\infty}\left(1+\left|\zeta_{1}\right|^{2}\right)^{-s} \mathrm{e}^{-\lambda \zeta_{1}} \mathrm{~d} \zeta_{1} \\
& \leq C \lambda^{2} \int_{0}^{\infty} \zeta_{1}^{-2 s} \mathrm{e}^{-2 \lambda \zeta_{1}} \mathrm{~d} \zeta_{1} \\
& \leq C \lambda \int_{0}^{\infty}\left(\frac{\zeta_{1}}{\lambda}\right)^{-2 s} \mathrm{e}^{-2 \zeta_{1}} \mathrm{~d} \zeta_{1} \leq C^{\prime} \lambda^{1+2 s} .
\end{aligned}
$$

With the same computation, we have the bound for $s<0$ (notice that it only holds in the homogeneous space $\dot{H}^{-s}$ )

$$
\left\|\frac{\lambda|\xi| \psi\left(P R_{\xi} v\right)}{\lambda|\xi|+i \xi \cdot v}\right\|_{\dot{H}_{v}^{-s}} \leq C \lambda^{1 / 2+s}
$$

Now for $s>1 / 2$, we trivially obtain

$$
\left\|\frac{\lambda|\xi| \psi\left(P R_{\xi} v\right)}{\lambda|\xi|+i \xi \cdot v}\right\|_{H_{v}^{-s}} \leq C \lambda
$$


because, assuming again that $\xi$ is parallel to the first coordinate axis,

$$
\left\|\frac{\lambda|\xi| \psi\left(P R_{\xi} v\right)}{\lambda|\xi|+i \xi \cdot v}\right\|_{H_{v}^{-s}}^{2} \leq C \lambda^{2} \int_{0}^{\infty} \frac{\mathrm{e}^{-2 \lambda \zeta_{1}}}{\left(1+\zeta_{1}^{2}\right)^{s}} \mathrm{~d} \zeta_{1} \leq C \lambda^{2} \int_{0}^{\infty} \frac{1}{\left(1+\zeta_{1}^{2}\right)^{s}} \mathrm{~d} \zeta_{1} \leq C \lambda^{2} .
$$

To conclude, for $s<0$, for any $f \in L_{x}^{2} H_{v}^{s}$, because of estimate (2.6)

$$
\begin{aligned}
\left\|T_{1}(f)\right\|_{L^{2}} & \leq\|f \phi\|_{L_{x}^{2} H_{v}^{s}}\left\|\frac{\lambda|\xi| \psi\left(P R_{\xi} v\right)}{\lambda|\xi|+i \xi \cdot v}\right\|_{L_{x}^{\infty} H_{v}^{-s}} \\
& \leq C\|f\|_{L_{x}^{2} H_{v}^{s}} \times\left(\left\|\frac{\lambda|\xi| \psi\left(P R_{\xi} v\right)}{\lambda|\xi|+i \xi \cdot v}\right\|_{L_{x}^{\infty} \dot{H}_{v}^{-s}}+\left\|\frac{\lambda|\xi| \psi\left(P R_{\xi} v\right)}{\lambda|\xi|+i \xi \cdot v}\right\|_{L_{x}^{\infty} L_{v}^{2}}\right) \\
& \leq C_{\phi}\left(\lambda^{1 / 2+s}+\lambda^{1 / 2}\right)\|f\|_{L_{x}^{2} H_{v}^{s} .}
\end{aligned}
$$

And for $s \geq 0$, for any $f \in L_{x}^{2} H_{v}^{s}$,

$$
\left\|T_{1}(f)\right\|_{L^{2}} \leq\|f \phi\|_{L_{x}^{2} H_{v}^{s}}\left\|\frac{\lambda|\xi| \psi\left(P R_{\xi} v\right)}{\lambda|\xi|+i \xi \cdot v}\right\|_{L_{x}^{\infty} H_{v}^{-s}} .
$$

The combination of this last inequality and of estimates (2.5) and (2.7) finishes the proof of the lemma (we recall that we work with compactly supported functions in $x$ ).

Remark. We do not know what is the best estimate for the critical exponent $s=1 / 2$. A variant of the previous proof gives

$$
\left\|T_{1}\right\|_{1 / 2,2} \leq-C \lambda \ln \lambda
$$

\subsection{Conclusion of the proof}

We now consider $g \in L_{x}^{p} W_{v}^{\beta, p}$ and $f \in L_{x}^{q} W_{v}^{\gamma, q}$ as given by (1.6) and satisfying equation (1.4).

We have obtained a decomposition of $\rho$ into a sum of two terms $T_{1}(f)$ and $T_{2}(g)$ in $L^{q}$ and in $W^{1-\alpha, p}$, depending on a parameter $\lambda$. We use this decomposition through Lions and Peetre's $K$-method of real interpolation (see in particular Lions and Peetre [18], Bergh and Löfström [2]). We define

$$
K(t, \rho)=\inf _{\substack{\rho_{1} \in L_{x}^{q}, \rho_{2} \in W_{x}^{1-\alpha, p} \\ \rho=\rho_{1}+\rho_{2}}}\left(\left\|\rho_{1}\right\|_{L^{q}}+t\left\|\rho_{2}\right\|_{W^{1-\alpha, p}}\right),
$$

and

$$
\Phi_{\theta, \infty}(\rho)=\left\|t^{-\theta} K(t, \rho)\right\|_{L_{t}^{\infty}} .
$$

By standard interpolation results, if $\Phi_{\theta, \infty}(\rho)<\infty$ then $\rho$ belongs to the interpolation space $\left(L^{q}, W^{1-\alpha, p}\right)_{\theta, \infty}$.

\subsubsection{The case of Theorem 1.1}

For $t>1$, then since we know that $\|\rho\|_{L^{q}} \leq C\|f\|_{L_{x, v}^{q}}$, we pose

$$
\rho_{1}=\rho, \quad \rho_{2}=0 .
$$

Therefore $t^{-\theta} K(t, \rho)$ is bounded uniformly for $t \in[1, \infty[$ for any $\theta \geq 0$.

For $t<1$ we use the natural decomposition given by the equation, i.e. we pose

$$
\rho_{1}=T_{1}(f), \quad \rho_{2}=T_{2}(g) .
$$


Of course it remains to choose $\lambda$ : thanks to Proposition 2.1, we have

$$
\left\|T_{1}(f)\right\|_{L^{q}}+t\left\|T_{2}(g)\right\|_{W^{1-\alpha, p}} \leq C \lambda^{r_{1}}+C t \lambda^{r_{2}},
$$

for any $r_{1}, r_{2}$ strictly less than $r_{1}^{*}, r_{2}^{*}$

$$
r_{1}^{*}=\gamma+1-\frac{1}{p}, \quad r_{2}^{*}=\beta-\frac{1}{q},
$$

because the parameters $\lambda$, which we will consider, are bounded from above, and thus we neglect the terms in $\lambda^{1-1 / p}$ or $\lambda^{1-1 / q}$ in the bounds for the case $\beta$ or $\gamma<0$. Minimizing in $\lambda$, we take

$$
\lambda=C t^{1 /\left(r_{1}+r_{2}\right)}
$$

and obtain for $t<1$ the estimate

$$
K(t, \rho) \leq C t^{\theta^{\prime}}
$$

for any $\theta^{\prime}$ strictly less than the exponent $\theta$ given by the formula (1.7).

We eventually deduce that $\rho$ belongs to the space $\left(L^{q}, W^{1-\alpha, p}\right)_{\theta^{\prime}, \infty}$ for any $\theta^{\prime}<\theta$ given by $(1.7)$.

This space is included in any $W_{\text {loc }}^{s^{\prime}, r}$ for $s^{\prime}<s=\theta(1-\alpha), 1 / r^{\prime}>1 / r=(1-\theta) / q+\theta / p$, thus proving Theorem 1.1. Indeed, locally in $x$, it is included in $\left(B_{q^{\prime} q^{\prime}}^{0}, B_{p^{\prime} p^{\prime}}^{1-\alpha}\right)_{\theta^{\prime}, r}$ for $q^{\prime}<q, p^{\prime}<p, \theta^{\prime}<\theta$.

\subsubsection{The case of Theorem 1.2}

The proof is exactly the same as for Theorem 1.1. But since we also know that $\beta$ and $\gamma$ are non negative or that $p=q=2$, we may take the limit values $r_{1}=r_{1}^{*}$ and $r_{2}=r_{2}^{*}$ and therefore we know that $\rho$ belongs to $\left(L^{q}, W^{1-\alpha, p}\right)_{\theta, \infty}$ with $\theta$ given by (1.7). This space turns out to be exactly the Besov space $B_{r \infty}^{s}$ if $p=q=r$.

\subsubsection{The case of Theorem 1.3}

The subcase $p \neq q$ in Theorem 1.3 is the more complicated because it is the only one where we have to use the decomposition (2.8) also for large $t$. By comparison the subcase $p=q$ in Theorem 1.3 is the easiest because we do not need the interpolation argument there and so we will not treat it (the argument would the same as in [24]).

For $\alpha=1$, and $0 \leq \beta, \gamma<1 / 2$, we define $\rho_{1}$ and $\rho_{2}$ by (2.8) for any $t$. Because of Proposition 2.1, we have for any $0<\delta_{1}<\gamma+1-1 / q, \delta_{2}<\beta-1 / p$, for any $\lambda>0$

$$
K(t, \rho) \leq C \lambda^{\delta_{1}}\|f\|_{L_{x}^{q} W_{v}^{\gamma, q}}+C t \lambda^{\delta_{2}}\|g\|_{L_{x}^{p} W_{v}^{\beta, p}} .
$$

Thus choosing $\lambda^{\delta_{1}-\delta_{2}}=t\|g\| /\|f\|$, we obtain

$$
K(t, \rho) \leq C t^{\delta_{1} /\left(\delta_{1}-\delta_{2}\right)}\|f\|_{L_{x}^{q} W_{v}^{\gamma, q}}^{1-\delta_{1} /\left(\delta_{1}-\delta_{2}\right)}\|g\|_{L_{x}^{p} W_{v}^{\beta, p}}^{\delta_{1} /\left(\delta_{1}-\delta_{2}\right)}
$$

The exponent $\delta_{1} /\left(\delta_{1}-\delta_{2}\right)$ being as close as we wish to (but less than) $\theta=(1+\gamma-1 / q) /(1+\gamma-1 / q-\beta+1 / p)$, we obtain $\rho$ in the spaces $\left(L^{p}, L^{q}\right)_{\theta^{\prime}, \infty}=L_{r \infty}$ (the Lorentz spaces) with $1 / r=\theta^{\prime} / p+\left(1-\theta^{\prime}\right) / q$ for any $\theta^{\prime}<\theta$ and with normless than $\left\|t^{-\theta^{\prime}} K(t, \rho)\right\|_{L_{t}^{\infty}}$. This exactly gives the corresponding inequality in Theorem 1.3 , thus concluding the proof.

As a last remark, notice that we cannot treat the case $\beta<0$ or $\gamma<0$ in Theorem 1.3, only because there the operator $T_{1}$ (or $T_{2}$ ) does not have the same behaviour in $\lambda$ for large and small $\lambda$.

\section{Proof of Proposition 2.1 And the $\mathcal{H}^{1}$ Bound}

Proposition 2.1 is obtained thanks to standard interpolation results between the $L^{2}$ case which has already been proved and a similar estimate in Hardy spaces. This requires some preliminary lemmas (Lems. 3.1, 3.3, 3.4 below). Combined with Lemma 2.1, they prove the proposition. 


\subsection{The product Hardy spaces}

The estimate on $T_{1}$ from $L_{x}^{p} W_{v}^{s, p}$ to $L^{p}$ for $p<2$ is obtained by interpolation. However as it is usual in this case, the operator $T_{1}$ is not bounded from $L_{x}^{1} W_{v}^{s, 1}$ to $L^{1}$ and as a consequence we need to work on some kind of Hardy space. Because the term $\xi \cdot v$ yields an operator which is not in Calderon-Zygmund class, we use here the product Hardy spaces which has been used in this context by Bézard in [4] and Bouchut [6].

We do not recall the definition of product Hardy spaces and refer the reader to [4] or [6] for details. What we only need is that these spaces, denoted $\mathcal{H}^{1}\left(\mathbb{R}^{d-1} \times \mathbb{R}, W^{s, 1}\right)$, satisfy the two following lemmas:

Lemma 3.1. (Interpolation) Let $T$ be bounded on

$$
\begin{gathered}
H_{v}^{s}\left(\mathbb{R}^{d}, L_{x}^{2}\left(\mathbb{R}^{d}\right)\right) \longrightarrow L_{x}^{2}\left(\mathbb{R}^{d}\right), ; \text { with norm }\|T\|_{s, 2}, \\
W_{v}^{s, 1}\left(\mathbb{R}^{d}, \mathcal{H}_{x}^{1}\left(\mathbb{R}^{d-1} \times \mathbb{R}\right)\right) \longrightarrow \mathcal{H}_{x}^{1}\left(\mathbb{R}^{d}\right), \text { with } \operatorname{norm} \mid T \|_{s, 1},
\end{gathered}
$$

then for any $1<p \leq 2, T$ is bounded on

$$
W_{v}^{s, p}\left(\mathbb{R}^{d}, E_{x}^{p}\left(\mathbb{R}^{d}\right)\right) \longrightarrow L_{x}^{p}\left(\mathbb{R}^{d}\right), \text { with norm } \mid T\left\|_{s, p} \leq\right\| T\left\|_{s, 1}^{2 / p-1}\right\| T \|_{s, 2}^{2-2 / p}
$$

This lemma is a direct consequence of Proposition 3.12 in [4] and of standard results on the interpolation of $L^{p}$ spaces (see [2]). Next we need a sufficient condition for a Fourier multiplier to be bounded on $\mathcal{H}^{1}\left(\mathbb{R}^{d-1} \times \mathbb{R}\right)$, which is exactly Proposition 3.10 in [4] or Lemma 1.8 in [6].

Lemma 3.2. Let $K\left(\xi^{\prime}, \xi_{d}\right) \in C^{\infty}\left(\mathbb{R}^{d} \backslash\left((0 \times \mathbb{R}) \cup\left(\mathbb{R}^{d-1} \times 0\right)\right)\right)$ such that for all $\alpha_{1} \in \mathbb{N}^{d-1}, \alpha_{2} \in \mathbb{N}$,

$$
\left|\partial_{\xi^{\prime}}^{\alpha_{1}} \partial_{\xi_{d}}^{\alpha_{2}} K\left(\xi^{\prime}, \xi_{d}\right)\right| \leq \frac{C_{\alpha_{1}, \alpha_{2}}}{\left|\xi^{\prime}\right|^{\alpha_{1}}\left|\xi_{d}\right|^{\alpha_{2}}}
$$

then $K$ is a bounded Fourier multiplier on $\mathcal{H}^{1}\left(R^{d-1} \times \mathbb{R}\right)$.

\subsection{Estimates for the norms of $T_{1}$ in $L^{p}$ spaces}

We now denote by $R_{v}$ the rotation in $\mathbb{R}^{d}$ such that $R_{v} v=|v| e_{d}$, where $e_{d}$ is the last coordinate vector. We perform the change of variable $x \rightarrow R_{v} x$ in definition (2.4) of $T_{1}$. We obtain that

$$
T_{1} f=\bar{T}_{1}\left(f\left(R_{v}^{-1} x, v\right)\right)
$$

with

$$
\bar{T}_{1}(f)=\mathcal{F}^{-1}\left(\int_{\mathbb{R}^{d}} \frac{\lambda|\xi|}{\lambda|\xi|+i \xi_{d}|v|} \hat{f}(\xi, v) \phi(v) \mathrm{d} v\right) .
$$

Obviously, the norms of $T_{1}$ from $W_{v}^{s, p}\left(\mathbb{R}^{d}, L_{x}^{p}\left(\mathbb{R}^{d}\right)\right)$ into $\left.L_{x}^{p}\left(\mathbb{R}^{d}\right)\right)$ are exactly the same as the norms of $\bar{T}_{1}$. For this last operator, with the help of Lemma 3.2 , we prove the

Lemma 3.3. $(s \leq 0) \bar{T}_{1}: W_{v}^{s, 1}\left(\mathbb{R}^{d}, \mathcal{H}^{1}\left(\mathbb{R}^{d-1} \times \mathbb{R}\right)\right) \longrightarrow \mathcal{H}^{1}\left(\mathbb{R}^{d}\right)$ with norm

$$
\left\|\bar{T}_{1}\right\|_{s, 1} \leq C\left(\lambda^{s}+1\right) .
$$

Proof. We cannot use here any Fourier transform in $v$, unlike in the proof of Lemma 2.1. Since we only know explicitly the $s$ derivative in $v$ of the kernel $\lambda|\xi| /\left(\lambda|\xi|+\xi_{d}|v|\right)$ for numbers $s$ which are integers, we also perform some interpolation here. 
For any $-s \in \mathbb{N}$, we have of course

$$
\lambda^{-s} \partial_{|v|}^{-s}\left(\frac{\lambda|\xi|}{\lambda|\xi|+i \xi_{d}|v|}\right)=(-s) ! \frac{\left(-i \xi_{d}\right)^{-s} \lambda^{1-s}|\xi|}{\left(\lambda|\xi|+i \xi_{d}|v|\right)^{1-s}} .
$$

It is easy to check that this last kernel satisfies the condition in Lemma 3.2 with constants $C_{\alpha_{1}, \alpha_{2}}$ independent of $\lambda$ and $v_{d}$. Hence for any nonpositive integers $s, \bar{T}$ is bounded from $W_{v}^{s, 1}\left(\mathbb{R}^{d}, \mathcal{H}^{1}\left(\mathbb{R}^{d-1} \times \mathbb{R}\right)\right)$ to $\mathcal{H}^{1}\left(\mathbb{R}^{d}\right)$ with norm

$$
\left\|\bar{T}_{1}\right\|_{s, 1} \leq C\left(\lambda^{s}+1\right) .
$$

Now we interpolate between the spaces $W_{v}^{-n, 1} \mathcal{H}_{x}^{1}\left(\mathbb{R}^{d-1} \times \mathbb{R}^{d}\right)$ and $W_{v}^{-n-1,1} \mathcal{H}_{x}^{1}\left(\mathbb{R}^{d-1} \times \mathbb{R}^{d}\right)$, we obtain all the spaces $W_{v}^{s, 1} \mathcal{H}^{1}\left(\mathbb{R}^{d-1} \times \mathbb{R}^{d}\right)$ for any $-n-1 \leq s \leq n$. So the last inequality is also true for any nonpositive $s$ and the lemma is proved.

We next consider the case where $f$ is more regular in $v$, i.e. $s>0$. Integrating by parts in $T_{1}$, we find with $K$ the diameter of the support of $\phi$

$$
\begin{aligned}
T_{1}(f)= & \mathcal{F}^{-1}\left(\int _ { \mathbb { R } ^ { d } } \frac { \lambda | \xi | } { \lambda | \xi | + i \xi \cdot v } \int _ { - \infty } ^ { 0 } \left(\phi\left(v+t \frac{\xi}{|\xi|}\right) \frac{\xi}{|\xi|} \cdot \nabla_{v} \hat{f}\left(\xi, v+t \frac{\xi}{|\xi|}\right)\right.\right. \\
& \left.\left.+\hat{f}\left(\xi, v+t \frac{\xi}{|\xi|}\right) \frac{\xi}{|\xi|} \cdot \nabla_{v} \phi\left(v+t \frac{\xi}{|\xi|}\right)\right) \mathrm{d} t \mathrm{~d} v\right) \\
T_{1}(f)= & \mathcal{F}^{-1}\left(\int _ { \mathbb { R } ^ { d } } ( \int _ { - 2 K } ^ { 0 } \frac { \lambda | \xi | \mathrm { d } t } { \lambda | \xi | + i \xi \cdot v - i t | \xi | } ) \left(\phi(v) \frac{\xi}{|\xi|} \cdot \nabla_{v} \hat{f}(\xi, v)\right.\right. \\
& \left.\left.+\hat{f}(\xi, v) \frac{\xi}{|\xi|} \cdot \nabla_{v} \phi(v)\right) \mathrm{d} v\right) .
\end{aligned}
$$

The function $\nabla_{v} \phi$ has exactly the same properties as $\phi$. After performing the change of variable $x \rightarrow R_{v} x$, we thus consider the following operator

$$
T_{1}^{\prime}(f)=\mathcal{F}^{-1}\left(\int_{\mathbb{R}^{d}}\left(\int_{-2 K}^{0} \frac{\lambda|\xi| \mathrm{d} t}{\lambda|\xi|+i \xi_{d}|v|-i t|\xi|}\right) \frac{\xi}{|\xi|} \hat{f}(\xi, v) \phi(v) \mathrm{d} v\right) .
$$

We prove that

Lemma 3.4. $T_{1}^{\prime}: L_{v}^{1}\left(\mathbb{R}^{d}, \mathcal{H}^{1}\left(\mathbb{R}^{d-1} \times \mathbb{R}\right)\right) \rightarrow \mathcal{H}^{1}\left(\mathbb{R}^{d}\right)$ with norm

$$
\left\|T_{1}^{\prime}\right\| \leq C \lambda \ln \lambda
$$

Proof. We only have to check that the kernel in the operator $T_{1}^{\prime}$ satisfies the conditions in Lemma 3.2. Indeed, we compute

$$
\begin{aligned}
\left.|| \xi^{\prime}\right|^{\alpha_{1}}\left|\xi_{d}\right|^{\alpha_{2}} \partial_{\xi^{\prime}}^{\alpha_{1}} \partial_{\xi_{d}}^{\alpha_{2}} & \int_{-2 K}^{0} \frac{\lambda|\xi| \mathrm{d} t}{\lambda|\xi|+i \xi_{d}|v|-i t|\xi|} \mid \leq \int_{-2 K}^{0} \frac{\lambda|\xi| \mathrm{d} t}{\lambda|\xi|+\left|\xi_{d}\right| v|-t| \xi||} \\
& \leq C \lambda \ln \lambda
\end{aligned}
$$

which proves the lemma. 


\section{The CASE OF HigheR REgUlarity IN VELOCITY}

In Theorems 1.1, 1.2 or 1.3 we have only considered functions $f$ or $g$ with less than half a derivative in velocity. From the proof of Proposition 2.1, it seems that formula (1.7) is not correct for $\beta$ or $\gamma$ larger than $1 / 2$ and should be modified according to the corresponding estimates in Proposition 2.1 for the operators. However, at least in the $L^{2}$ case, such a method does not lead to an optimal result. Notice also that when $\beta>1 / 2$ with $p=2$ (i.e. $g$ is very regular in velocity), Theorem 1.1 already gives the best regularity we may hope for, because $\theta=1$ and so we gain a full $1-\alpha$ derivative for the average. Throughout all this section, we thus consider the cases $p=q=2, \beta<1 / 2$ and $\gamma>1 / 2$ in (1.6).

In fact, in such a situation, the operator decomposition leading to Proposition 2.1 is unable to take advantage of the additional regularity of $f$ and Theorem 1.1 gives the same result as for $f \in L_{x}^{2} H_{v}^{1 / 2}$.

Using another decomposition than in Section 2, we can prove the

Theorem 4.1. Let $0 \leq \alpha<1$, let $f, g$ satisfy(1.4) and (1.6) with $p=q=2$ and $\beta<1 / 2$, then for $s=\theta(1-\alpha)$,

$$
\begin{gathered}
\|\rho\|_{H_{\mathrm{loc}}^{s}} \leq C\left(\|g\|_{L_{x}^{2} H_{v}^{\beta}}+\|f\|_{L_{x}^{2} H_{v}^{\gamma}}\right) \\
\text { with } \quad \theta=\frac{1 / 2+\gamma}{1+\gamma-\beta} .
\end{gathered}
$$

The values of $\theta$ given in (4.1) and in(1.7) with $p=q=2$ are the same. The new point here is the extension to values $\gamma>1 / 2$.

Proof of Theorem 4.1. We introduce a new decomposition of the average which depends on the point $\xi$ considered in the Fourier space.

For the moment, we keep this point fixed and we may thus assume that $\xi=(|\xi|, 0, \ldots, 0)$. Equation $(2.1)$ becomes simply

$$
i v_{1} \hat{f}=|\xi|^{\alpha-1} \hat{g}
$$

We denote by $\tilde{f}$ and $\tilde{g}$ the Fourier transform of $f$ and $g$ in the $x$ and $v$ variables and by $\eta$ the dual variable of $v_{1}$, and by $\eta^{\prime}$ the dual variable of $v^{\prime}=\left(v_{2}, \ldots, v_{d}\right)$. We then have for any $0<\lambda \leq 1$ (here we only need the case $\lambda$ small) and for an even exponent $k$ larger than $\gamma-1 / 2$

$$
-\lambda \eta^{k} \tilde{f}+\partial_{\eta} \tilde{f}=-\lambda \eta^{k} \tilde{f}+|\xi|^{\alpha-1} \tilde{g}
$$

We therefore obtain a decomposition (compare with (2.2))

$$
\hat{\rho}=|\xi|^{\alpha-1} \tilde{T}_{\xi} \hat{g}+\lambda \tilde{T}_{\xi}\left(\partial_{v_{1}}^{k} \hat{f}\right)
$$

with

$$
\tilde{T}_{\xi}^{k}(h(v))=\int_{\mathbb{R}^{d}} \mathcal{F}_{v_{1}} \phi\left(\eta, \eta^{\prime}\right) \int_{\eta}^{\infty} \mathcal{F}_{v} h\left(\mu, \eta^{\prime}\right) \mathrm{e}^{-\frac{\lambda}{k+1}\left(\mu^{k+1}-\eta^{k+1}\right)} \mathrm{d} \mu \mathrm{d} \eta \mathrm{d} \eta^{\prime}
$$

The operator $\tilde{T}_{\xi}^{k}$ is as a matter of fact a linear form, the variable $\xi$ being only a parameter, with the following estimate:

Lemma 4.1. for any real number $s<1 / 2, \tilde{T}_{\xi}^{k}: H_{v_{1}}^{s} \rightarrow \mathbb{R}$ with norm

$$
\left\|\tilde{T}_{\xi}^{k}\right\|_{s, 2} \leq C \lambda^{(s-1 / 2) /(k+1)}
$$


Proof. It is a direct computation from formula (4.3). Since the function $\phi$ has compact support and belongs to any $H^{s^{\prime}}$, we have, first for $s \geq 0$

$$
\begin{aligned}
\left|\tilde{T}_{\xi}^{k} h\right|^{2} \leq & C\|\phi\|_{L_{v^{\prime}}^{2} H_{v_{1}}^{1}} \times \int_{\mathbb{R}^{d-1}} \sup _{\eta}\left(\int_{\eta}^{\infty} \mathcal{F}_{v} h\left(\mu, \eta^{\prime}\right) \mathrm{e}^{-\frac{\lambda}{k+1}\left(\mu^{k+1}-\eta^{k+1}\right)} \mathrm{d} \mu\right)^{2} \mathrm{~d} \eta^{\prime} \\
\leq & C \int_{\mathbb{R}^{d-1}} \sup _{\eta}\left(\int_{\eta}^{\infty}|\mu|^{2 s}\left|\mathcal{F}_{v} h\left(\mu, \eta^{\prime}\right)\right|^{2} \mathrm{~d} \mu\right) \\
& \times\left(\int_{\eta}^{\infty}|\mu|^{2 s} \mathrm{e}^{-\frac{2 \lambda}{k+1}\left(\mu^{k+1}-\eta^{k+1}\right)} \mathrm{d} \mu\right) \mathrm{d} \eta^{\prime} \\
\leq & C\left(\int_{\mathbb{R}^{d}}|\mu|^{2 s}\left|\mathcal{F}_{v} h\left(\mu, \eta^{\prime}\right)\right|^{2} \mathrm{~d} \mu \mathrm{d} \eta^{\prime}\right) \\
& \times \lambda^{(2 s-1) /(k+1)} \sup _{\eta} \int_{\lambda^{1 /(k+1)} \eta}^{\infty}|\mu|^{-2 s} \mathrm{e}^{-\frac{2}{k+1}\left(\mu^{k+1}-\eta^{k+1}\right)} \mathrm{d} \mu \\
\leq & C \lambda^{(2 s-1) /(k+1)}\|h\|_{H^{s}}^{2} .
\end{aligned}
$$

For the case $s<0$, a similar computation leads to

$$
\left|\tilde{T}_{\xi}^{k} h\right|^{2} \leq C \lambda^{-1 /(k+1)}\left(1+\lambda^{2 s /(k+1)}\right)\|h\|_{H^{s}}^{2} \leq C \lambda^{(2 s-1) /(k+1)}\|h\|_{H^{s}}^{2},
$$

since we only consider parameters $\lambda \leq 1$.

The function $\partial_{v_{1}}^{k} \hat{f}$ belongs to $L_{\xi}^{2} H_{v}^{\gamma-k}$. Since $k>\gamma-1 / 2$, we may apply Lemma 4.1 and find

$$
\hat{\rho}(\xi)=C|\xi|^{\alpha-1} \lambda^{(\beta-1 / 2) /(k+1)}\|\hat{g}(\xi, .)\|_{H_{v}^{\beta}}+C \lambda^{(\gamma+1 / 2) /(k+1)}\|\hat{f}(\xi, .)\|_{H_{v}^{\gamma}} .
$$

We minimize in $\lambda$. The aim is of course to control $\hat{\rho}(\xi)$ for large $\xi$. Also notice that because of the localization in $x$, we may take $f$ and $g$ in $L_{x}^{1} H_{v}^{\gamma}$ and $L_{x}^{1} H_{v}^{\beta}$ with consequently $\hat{f}$ and $\hat{g}$ in $C_{\xi}^{0} H_{v}^{\gamma}$ and $C^{0} H_{v}^{\beta}$. Since $\alpha<1$, it is enough to consider parameters $\lambda \leq 1$ and we obtain

$$
\hat{\rho}(\xi) \leq C|\xi|^{(\alpha-1) \frac{1 / 2+\gamma}{1+\gamma-\beta}}\left(\|\hat{g}(\xi, .)\|_{H_{v}^{\beta}}+\|\hat{f}(\xi, .)\|_{H_{v}^{\gamma}}\right) .
$$

And finally

with $s$ given by Theorem 4.1 .

$$
\|\rho\|_{H_{l o c}^{s}} \leq C\left(\|g\|_{L_{x}^{2} H_{v}^{\beta}}+\|f\|_{L_{x}^{2} H_{v}^{\gamma}}\right)
$$

\section{REFERENCES}

[1] L. Ambrosio, C. De Lellis and C. Mantegazza, Line energies for gradient vector fields in the plane. Calc. Var. Partial Differential Equations 9 (1999) 327-355.

[2] J. Bergh and J. Löfström, Interpolation spaces, an introduction. Springer-Verlag, A Ser. of Comprehensive Stud. in Math. 223 (1976).

[3] Y. Brenier and L. Corrias, A kinetic formulation formulti-branch entropy solutions of scalar conservation laws. Ann. Inst. H. Poincaré Anal. Non Linéaire 15 (1998) 169-190.

[4] M. Bézard, Régularité $L^{p}$ précisée des moyennes dans les équations de transport. Bull. Soc. Math. France 122 (1994) 29-76.

[5] F. Bouchut and L. Desvillettes, Averaging lemmas without time Fourier transform and applications to discretized kineticequations. Proc. Roy. Soc. Edinburgh Ser. A 129 (1999) 19-36.

[6] F. Bouchut, F. Golse and M. Pulvirenti, Kinetic equations and asymptotic theory. Gauthiers-Villars, Ser. in Appl. Math. (2000).

[7] A. Desimone, R.W. Kohn, S. Müller and F. Otto, Magnetic microstructures, a paradigm of multiscale problems. Proc. of ICIAM (to appear). 
[8] R. DeVore and G.P. Petrova, The averaging lemma. J. Amer. Math. Soc. 14 (2001) 279-296.

[9] R. DiPerna and P.L. Lions, Global weak solutions of Vlasov-Maxwell systems. Comm. Pure Appl. Math. 42 (1989) $729-757$.

[10] R. DiPerna, P.L. Lions and Y. Meyer, $L^{p}$ regularity of velocity averages. Ann. Inst. H. Poincaré Anal. Non Linéaire 8 (1991) 271-287.

[11] P. Gérard, Microlocal defect measures. Comm. Partial Differential Equations 16 (1991) 1761-1794.

[12] F. Golse, Quelques résultats de moyennisation pour les équations aux dérivées partielles. Rend. Sem. Mat. Univ. Pol. Torino, Fascicolo Speciale 1988 Hyperbolic equations (1987) 101-123.

[13] F. Golse, P.L. Lions, B. Perthame and R. Sentis, Regularity of the moments of the solution of a transport equation. J. Funct. Anal. 26 (1988) 110-125.

[14] F. Golse, B. Perthame and R. Sentis, Un résultat de compacité pour les équations de transport et application au calcul de la limite de la valeur propre principale d'un opérateur de transport. C. R. Acad. Sci. Paris Sér. I Math. 301 (1985) 341-344.

[15] S. Hwang and A. Tzavaras, Kinetic decomposition of approximate solutions to conservation laws: Applications to relaxation and diffusion-dispersion approximations, Preprint. University of Wisconsin, Madison (2001).

[16] P.-E. Jabin and B. Perthame, Compactness in Ginzburg-Landau energy by kinetic averaging. Comm. Pure Appl. Math. 54 (2001) 1096-1109.

[17] P.-E. Jabin, F. Otto and B. Perthame, Line-energy Ginzburg-Landau models: Zero-energy states. Ann. Sc. Norm. Sup. Pisa (to appear).

[18] J.-L. Lions and J. Peetre, Sur une classe d'espaces d'interpolation. Inst. Hautes Études Sci. Publ. Math. 19 (1964) 5-68.

[19] P.L. Lions, Régularité optimale des moyennes en vitesse. C. R. Acad. Sci. Sér. I Math. 320 (1995) 911-915.

[20] P.L. Lions, B. Perthame and E. Tadmor, A kinetic formulation of multidimensional scalar conservation laws and related questions. J. Amer. Math. Soc. 7 (1994) 169-191.

[21] P.L. Lions, B. Perthame and E. Tadmor, Kinetic formulation of the isentropic gas dynamics and $p$-systems. Comm. Math. Phys. 163 (1994) 415-431.

[22] O.A. Oleı̆nik, On Cauchy's problem for nonlinear equations in a class of discontinuous functions. Doklady Akad. Nauk SSSR (N.S.) 95 (1954) 451-454.

[23] B. Perthame, Kinetic Formulations of conservation laws. Oxford University Press, Oxford Ser. in Math. and Its Appl. (2002).

[24] B. Perthame and P.E. Souganidis, A limiting case for velocity averaging. Ann. Sci. École Norm. Sup. (4) 31 (1998) 591-598.

[25] M. Porthileiro, Compactness of velocity averages. Preprint.

[26] T. Rivière and S. Serfaty, Compactness, kinetic formulation, and entropies for a problem related to micromagnetics. Preprint (2001).

[27] A. Vasseur, Time regularity for the system of isentropic gas dynamics with $\gamma=3$. Comm. Partial Differential Equations 24 (1999) 1987-1997.

[28] M. Westdickenberg, some new velocity averaging results. SIAM J. Math. Anal. (to appear).

[29] C. Cheverry, Regularizing effects for multidimensional scalar conservation laws. Ann. Inst. H. Poincaré Anal. Non Linéaire 17 (2000) 413-472. 